\title{
Qualidade de vida de indivíduos pós acidente vascular cerebral: uma revisão integrativa
}

\author{
Quality of life of individuals after stroke: an integrative review \\ Calidad de vida de los individuos después del accidente cerebrovascular: una revisión integradora
}

Recebido: 03/11/2021 | Revisado: 08/11/2021 | Aceito: 08/11/2021 | Publicado: 12/11/2021

\author{
Marcos Linco Conceição \\ ORCID: https://orcid.org/0000-0003-1525-5834 \\ Centro Universitário da Amazônia, Brasil \\ E-mail: linco.marcos91@gmail.com \\ Marcelo Linco Conceição \\ ORCID: https://orcid.org/0000-0001-7127-5376 \\ Centro Universitário da Amazônia, Brasil \\ E-mail: mlinco50@gmail.com \\ Paulo Henrique Ramos Pimentel \\ ORCID: https://orcid.org/0000-0002-9561-5715 \\ Centro Universitário da Amazônia, Brasil \\ E-mail: pauloramosfisio@ hotmail.com
}

\begin{abstract}
Resumo
Introdução: O AVC é considerado uma das questões de saúde pública no mundo inteiro, se tornando a segunda causa no ranking de mortalidade mais comuns nos países, sendo uma das principais causas de incapacidade física temporária ou definitiva. Objetivo: Investigar na literatura existente as repercussões do Acidente Vascular Cerebral (AVC) na qualidade de vida dos indivíduos. Metodologia: Trata-se de uma revisão integrativa da literatura, incluindo as bases de dados da LILACS e MEDLINE, PUBMED e PeDro, Acervo+ Index Base e SciELO, na qual a amostra final para compor está revisão integrativa, foram selecionados onze artigos a partir dos critérios de inclusão e exclusão. Resultados: Identificou-se que a qualidade de vida é o principal elemento afetado em decorrência do AVC, segundo o SF-36, destacando-se os aspectos físicos e emocionais, como sendo os mais afetados e os que mais geram complicações na QV de pacientes diagnosticados com AVC. Considerações finais: O presente estudo possibilitou analisar a QV dos indivíduos e os principais elementos afetados devido aos diversos problemas obtidos em decorrência do AVC, foi possível observar os impactos causados pelo AVC na QV dos indivíduos acometidos, acarretando complicações nos aspectos relacionados a saúde física e emocional principalmente.
\end{abstract}

Palavras-chave: Fisioterapia; AVC; Qualidade de vida.

\begin{abstract}
Introduction: Stroke is considered one of the public health issues worldwide, becoming the second most common cause in mortality in countries, being one of the main causes of temporary or permanent physical disability. Objective: To investigate in the existing literature the repercussions of stroke in the quality of life of individuals. Methodology: This is an integrative review of the literature, including the databases of LILACS and MEDLINE, PUBMED and PeDro, Acervo+ Index Base e SciELO, in which the final sample to be compose this integrative review, eleven articles were selected based on the inclusion and exclusion criteria. Results: It was identified that quality of life is the main element affected due to stroke, according to the SF-36, the physical and emotional aspects stand out, as being the most affected and the ones that most generate complications in the quality of life of patients diagnosed with stroke. Final considerations: The present study made it possible to analyse the quality of life of individuals and the main elements affected due to the various problems obtained as a result the stroke on the quality of life of affected individuals, resulting in complications in aspects related to physical and emotional health mainly.
\end{abstract}

Keywords: Physiotherapy; Stroke; Quality of life.

\section{Resumen}

Introducción: El ictus es considerado uno de los problemas de salud pública a nivel mundial, convirtiéndose en la segunda causa más común de mortalidad en los países, siendo una de las principales causas de discapacidad física temporal o permanente. Objetivo: Investigar en la literatura existente las repercusiones del ictus en la calidad de vida de los individuos. Metodologia: Esta es una revisión integradora de la literatura, incluyendo las bases de datos de LILACS y MEDLINE, PUBMED y PeDro, Acervo+ Index Base e SciELO, en la que la muestra final a componer es la revisión integradora, se seleccionaron once artículos en base a los criterios de inclusión y exclusión. Resultados: Se 
identificó que la calidad de vida es el principal elemento afectado por accidente cerebrovascular, según el SF-36, destacan los aspectos físicos y emocionales, por ser los más afectados y los que más generan complicaciones en la calidad de vida de los pacientes diagnosticados con ictus. Consideraciones finales: El presente estudio permitió analizar la calidad de vida de los individuos y de los principales elementos afectados debido a los diversos problemas obtenidos como consecuencia de lo ictus en la calidad de vida de los individuos afectados, resultando en complicaciones en aspectos relacionados con la salud física y emocional principalmente.

Palabras clave: Fisioterapia; AVC; Calidad de vida.

\section{Introdução}

O AVC é considerado uma das questões de saúde pública no mundo inteiro, se tornando a segunda causa no ranking de mortalidade mais comuns nos países, sendo uma das principais causas de incapacidade física temporária ou definitiva, causador do número elevado de internações (Santos \& Waters, 2020). O AVC é resultante de uma obstrução ou ruptura da circulação sanguínea em uma determinada parte do cérebro, podendo ser subdivido em AVC Isquêmico e AVC Hemorrágico, onde o primeiro, ocorre uma interrupção nos vasos sanguíneos que irrigam o cérebro, e o segundo, por sua vez, caracteriza-se pelo rompimento dos vasos cerebrais, causando um extravasamento de sangue. Os dois tipos geram uma diminuição no suprimento sanguíneo, levando à morte de células nervosas (Coradini et al., 2020; Mello et al., 2020).

Silva et al. (2019) apontam que o índice de morte associada ao AVC, depende da localidade, sendo diretamente relacionada pela qualidade das políticas públicas, correlacionadas às medidas preventivas quanto terapêuticas das doenças cerebrovasculares. Estudos estabelecem, que as maiores taxas de mortalidade pelo AVC encontram-se nos países subdesenvolvidos ou em desenvolvimento e, em âmbito nacional, costumam ser maiores nos estados mais pobres e menos desenvolvidos socioeconomicamente (Silva et al., 2019).

Além de ser uma das principais causas de óbitos no Brasil, a literatura aponta que aproximadamente $70 \%$ dos pacientes que são acometidos com AVC acabam ficando incapacitados em retornar às suas atividades laborais e, 50\% deles, apresentam dificuldades até nas suas Atividades de Vida Diárias (AVD’s). Essas incapacidades, acabam gerando notáveis complicações no bem-estar físico, mental e social do indivíduo (Carvalho et al., 2019).

Diante das consequências adquiridas pelo AVC, é possível notar que após um acometimento, a saúde física e emocional dessas pessoas é a mais atingida, pois quando não leva a óbito deixa o indivíduo com certas incapacidades, dependendo da gravidade e do nível do acometimento, sendo a qualidade de vida (QV) um principal determinante da saúde. A Organização Mundial da Saúde (OMS) (2012), define a QV como a interpretação por meio da percepção do próprio indivíduo, ou seja, no seu contexto cultural, com relação aos seus objetivos, expectativas, padrões e preocupações.

Nessa perspectiva, existem instrumentos que são utilizados para verificar a QV de um determinado grupo que deseja observar, dentre eles, está o Short Form-36 (SF-36), que tem como característica em analisar a QV de pessoas que apresentam algumas complicações de saúde (Yang et al., 2019).

O SF-36 trata-se de um questionário validado com a finalidade de avaliar a QV. O instrumento é composto por oito domínios, sendo eles: funcionamento físico (FP); papel-físico (RP), ou limitação no funcionamento diário do papel devido a problemas físicos; papel emocional (RE), ou limitação no funcionamento diário do papel devido a problemas emocionais; dor corporal (PA); saúde geral percepção (GH); vitalidade (VT); funcionamento social (SF); e percepção da saúde mental (SM). Cada escala consiste em 2 a 10 itens, e cada item é classificado em um Likert de dois a seis pontos escala, a pontuação da escala é calculada pela soma de todos os itens (Yang et al., 2019).

Dado o exposto, a QV é o principal elemento afetado devido aos diversos problemas obtidos em decorrência de um AVC. Portanto, o objetivo do presente estudo é investigar na literatura existente as repercussões do Acidente Vascular Cerebral na qualidade de vida dos indivíduos. 


\section{Metodologia}

Trata-se de revisão integrativa de literatura, desenvolvida no período de setembro de 2021, visando o aprofundamento da temática em questão através da reunião e síntese de achados de estudos já realizados (Soares et al., 2014). Optou-se por utilizar estudos que se enquadrarem nos seguintes critérios de inclusão: estudos com período determinado de 10 anos (2011 a 2021), que apresentem em seu conteúdo relação direta com o objetivo da pesquisa, em língua portuguesa e/ou inglesa. Foram excluídos estudos que não estivessem disponíveis o acesso de sua versão completa, estudos sem correlação sobre o assunto, ou que não se enquadrarem aos critérios de inclusão.

Essa revisão integrativa utilizou 4 etapas, sendo elas: 1) busca na literatura; 2) avaliação dos estudos elegíveis; 3) análise e interpretação dos resultados; 4) elaboração da revisão (Soares et al., 2014).

\section{Etapa 1 - Busca na Literatura}

Os estudos utilizados para compor esta revisão integrativa, encontram-se indexados nas seguintes bases de dados eletrônicas: PUBMED, Literatura Latino-americana em Ciências da Saúde (LILACS), Medical Literature Analyses and System Online (MEDLINE), PeDro, Acervo+ Index Base e Scientific Electronic Library Online (SciELO). Para a busca dos estudos foram escolhidos os seguintes Descritores em Ciências da Saúde (DeCS): Fisioterapia, AVC, Qualidade de vida, SF-36, e seus correspondentes na língua inglesa: Physiotherapy, Stroke, Quality of life. Em seguida foram realizadas as análises e as seleções dos estudos que fizeram parte da revisão segundo os critérios de inclusão e exclusão (Figura 1).

\section{Etapa 2 - Avaliação dos estudos elegíveis}

Todos os artigos selecionados foram lidos integralmente, e posteriormente passaram pelo processo de triagem e os elegíveis foram realizados as avaliações e as organizações dos dados, após a leitura do material selecionado as informações necessárias foram extraídas e anexadas no quadro abaixo (Quadro 1). Com a seguinte sequência: n, autores (ano), intervenção e principais achados.

\section{Etapa 3 - Análise e interpretação dos resultados}

A análise e interpretação dos dados, nesta etapa foram feitas as interpretações e as discussões dos resultados obtidos por meio das informações adquiridos através dos estudos, com a finalidade de chegar na resposta da pesquisa em questão.

\section{Etapa 4 - Elaboração da revisão}

O resultado desta etapa consistiu na elaboração da revisão para a construção final do estudo, sendo produto da análise dos dados. 
Figura 1 - Fluxograma do processo de seleção dos artigos da revisão, segundo PRISMA.
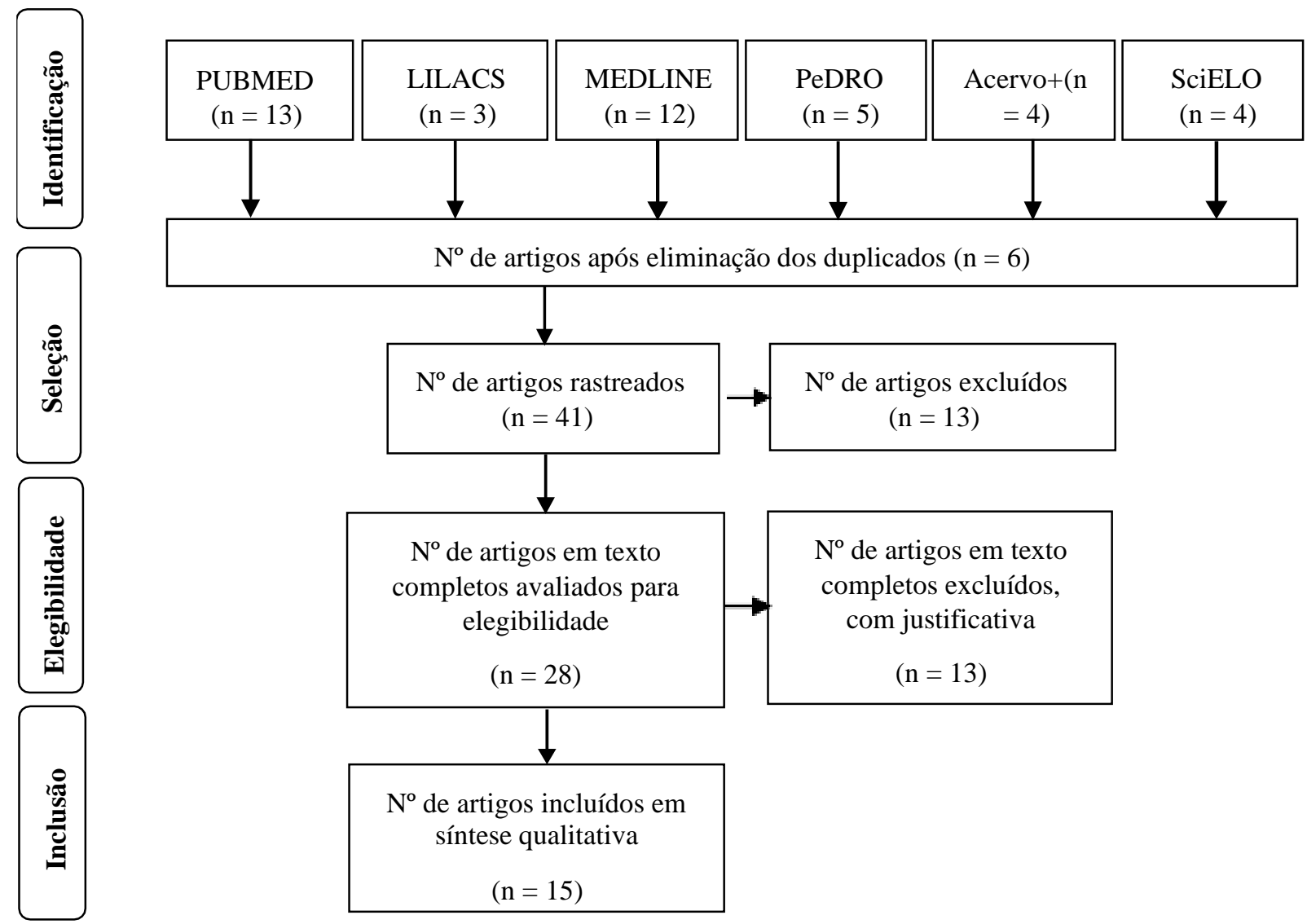

Fonte: Conceição, Conceição e Pimentel (2021).

\section{Resultados}

O (Quadro 1) a seguir apresenta as informações extraídas dos quinze estudos selecionados, sendo numerados de 1 a 15, identificando os autores e ano, bem como as intervenções do estudo e seus principais achados.

Quadro 1 - Os principais achados dos artigos selecionados para a revisão integrativa da literatura.

\begin{tabular}{|c|c|c|c|}
\hline $\mathbf{N}$ & Autores (ano) & Intervenção & Principais achados \\
\hline 1 & $\begin{array}{l}\text { Dezincourt et al., } \\
\text { (2021). }\end{array}$ & $\begin{array}{l}\text { Trata-se de um relato de experiência, conduzido com } \\
\text { uma paciente cadastrada em uma Unidade Básica de } \\
\text { Saúde (UBS) no município de Santarém-PA, nos } \\
\text { meses de outubro e dezembro de } 2019 \text {. }\end{array}$ & $\begin{array}{l}\text { Notou-se melhora na coordenação e aparente } \\
\text { relaxamento da musculatura, além de um } \\
\text { aumento da motivação, autoestima, bem como } \\
\text { da reintegração das AVD'S. }\end{array}$ \\
\hline 2 & $\begin{array}{l}\text { Assis et al., } \\
\text { (2021). }\end{array}$ & $\begin{array}{l}\text { Estudo retrospectivo de corte transversal, dos } \\
\text { pacientes admitidos consecutivamente na Fundação } \\
\text { Baiana de Neurologia de } 2017 \text { a } 2019 \text { com o } \\
\text { diagnóstico de AVC isquêmico agudo. Analisamos os } \\
\text { subtipos do AVC utilizando a classificação do } \\
\text { Oxfordshire Community Stroke Project. }\end{array}$ & $\begin{array}{l}\text { Constataram que a média da idade dos } \\
\text { pacientes foi semelhante entre os diversos } \\
\text { subtipos do AVC. Observamos uma alta } \\
\text { frequência de hipertensão arterial, entretanto, } \\
\text { não encontramos associação desta condição } \\
\text { com nenhum dos subtipos. }\end{array}$ \\
\hline 3 & $\begin{array}{l}\text { Barbosa et al., } \\
\text { (2021). }\end{array}$ & $\begin{array}{l}\text { Este estudo se baseou nas informações contidas nos } \\
\text { bancos de dados do Sistema de Informações } \\
\text { Hospitalares (SIH) e no Departamento de Informática } \\
\text { do Sistema Único de Saúde (DATASUS) dos casos } \\
\text { de AVC nos anos de } 2010 \text { a } 2019 \text {. }\end{array}$ & $\begin{array}{l}\text { Os resultados revelaram um aumento gradativo } \\
\text { da doença durante o período analisado. Quanto } \\
\text { à mortalidade da doença, não houve alterações } \\
\text { bruscas no período estudado, observando-se } \\
\text { uma média de } 17,74 \% \text { de mortes por casos. }\end{array}$ \\
\hline 4 & $\begin{array}{l}\text { Cruz et al., } \\
(2020) .\end{array}$ & $\begin{array}{l}\text { A escala de escala analógica visual para dor, a escala } \\
\text { de resiliência e a escala de qualidade de vida SF-36 } \\
\text { foi utilizada como medida de desfecho. }\end{array}$ & $\begin{array}{l}\text { O exercício físico realizado na água tem efeitos } \\
\text { positivos em vários fatores que contribuem } \\
\text { para melhor o humor e a QV das pessoas com } \\
\text { lesão cerebral adquirida. }\end{array}$ \\
\hline 5 & $\begin{array}{l}\text { Gonçalves et al., } \\
\text { (2018). }\end{array}$ & $\begin{array}{l}\text { Foram estudados } 18 \text { pacientes ( } 8 \text { mulheres, } 55,5 \pm \\
13,9 \text { anos). Fizeram uso da Realidade Virtual }(\mathrm{RV})\end{array}$ & $\begin{array}{l}\text { Os achados obtidos mostraram que a } \mathrm{QV} \text {, } \\
\text { independência funcional, características }\end{array}$ \\
\hline
\end{tabular}




\begin{tabular}{|c|c|c|}
\hline & & $\begin{array}{l}\text { associado à reabilitação convencional na função do } \\
\text { membro superior após o AVC. }\end{array}$ \\
\hline 6 & $\begin{array}{l}\text { Eyvaz et al., } \\
\text { (2018). }\end{array}$ & $\begin{array}{l}\text { Terapia WBE }(3 / \text { semana })+\text { a combinação LBE }(2 / \\
\text { semana }) \text { foi aplicada aos pacientes do grupo de } \\
\text { estudo }(\mathrm{n}=30) \text { por seis semanas. LBE era aplicado } \\
\text { ao grupo controle }(\mathrm{n}=30) 5 / \text { semana por seis } \\
\text { semanas. Foram avaliados antes e após o tratamento. }\end{array}$ \\
\hline 7 & $\begin{array}{l}\text { Kiingi et al., } \\
\text { (2017). }\end{array}$ & $\begin{array}{l}\text { Este foi um estudo transversal usando as seguintes } \\
\text { medidas de desfecho: Medida de reintegração da } \\
\text { comunidade de AVC, escala de QV específica para } \\
\text { AVC, cuidador índice de deformação e questionário } \\
\text { de satisfação do paciente em fisioterapia. }\end{array}$ \\
\hline
\end{tabular}

8 Carregosa et al., Para a avaliação, a Pesquisa de Saúde de Forma Curta (2017). 36 (SF-36) escalas foram utilizados. Os pacientes foram imediatamente avaliados após a conclusão de a intervenção e 2 meses após a segunda avaliação.

$9 \quad$ Liria et al., (2016).

10 Ribeiro et al., (2015).

Grupo RITH foram tratados por uma equipe que incluiu um fisioterapeuta, um terapeuta ocupacional e um médico de reabilitação. Grupo hospitalar foram tratados de acordo com a reabilitação padrão dos procedimentos na Unidade Hospitalar de AVC por fisioterapeutas.

(2015)

Os pacientes foram submetidos a sessões de tratamento de 60 minutos duas vezes por semana durante dois meses. $\mathrm{O}$ grupo que passou por reabilitação virtual, fez em uma sala equipada com o NW e um projetor multimídia.

11 Kim et al., (2015). Foram tratados com fisioterapia geral e Tai Chi exercício (11 pacientes) e um tratado apenas com fisioterapia (11 pacientes). Tai Chi terapêutico incluído 10 movimentos diferentes e foram realizados por $60 \mathrm{~min}$, duas vezes por semana, durante 6 semanas.

12 Gordon et al., (2015).

O grupo de intervenção $(n=64)$ caminhou sobre o solo por 30 minutos, 3 vezes por semana durante 12 semanas. $O$ grupo controle $(n=64)$ recebeu massagem no lado afetado.

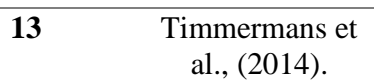

Assistidos por robô (grupo experimental) ou nãorobótico orientado para a tarefa treinamento braçomão (grupo controle). O treinamento foi fornecido durante 8 semanas, 4 vezes / semana, $2 \times 30 \mathrm{~min} /$ dia.

14 Dundar et al., (2014).

15 Beinotti et al., (2013).
O CP programa focado na facilitação de movimentos, faixa de movimento, alongamento exercícios, fortalecimento de membros superiores e inferiores exercícios, e melhorar o equilíbrio, de pé, sentado, transferindo e andando.

O grupo de controle participou de um programa de fisioterapia convencional, enquanto o grupo experimental participou de fisioterapia mais sessões de HBRT por 16 semanas. funcionais e destreza do membro superior apresentaram melhora após a RV.

Os resultados pós-tratamento mostraram melhorias significativas em todos os parâmetros (exceto SF-36 parâmetro de dor) em ambos os grupos. A melhora no parâmetro de vitalidade do SF-36 foi maior no grupo de estudo.

A QV foi ruim com as pontuações mais baixas para energia e pontuações mais altas para os domínios da visão e da linguagem. Foi encontrada uma correlação positiva entre reintegração na comunidade e satisfação com serviços.

Após a terapia NW, os pacientes tiveram aprendizado motor retenção, obtendo um benefício sustentado através da técnica e mostraram melhora na QV nos aspectos de capacidades funcionais.

Após a fisioterapia, ambos os grupos mostraram melhorias estatisticamente significativas desde a linha de base em cada uma das medidas. Essas melhorias foram melhores em pacientes RITH do que em pacientes hospitalares, em todas as escalas de funcionalidade com menos sessões.

O SF-36 revelou uma diferença significativa dentro de ambos os grupos em relação aos seguintes domínios: funcionamento físico, limitação de funções devido a aspectos físicos, vitalidade e limitação de funções devido a aspectos emocionais.

Na QV, o grupo terapêutico de Tai Chi mostrou uma melhora significativa em cinco itens (função física, dor, vitalidade, saúde geral, saúde mental) entre os oito itens do SF36.

Houve uma tendência de maior melhoria ao longo do tempo para o componente de saúde física do SF-36 e melhora significativamente maior ao longo do tempo para a distância percorrida em 6 minutos em favor da caminhada grupo.

Uma melhora significativa no componente de saúde física SF-36 foi encontrada no grupo experimental, entre o início e a interrupção do treinamento ( $\mathrm{SF}-36$ saúde física, $\mathrm{p}=0,01$ ).

A TR combinada com o CP produziu melhoria nas subpartes de SF-36 dos pacientes com AVC subagudo e crônico (até 1 ano) do que o programa CP.

Foi observada melhora significativa na pontuação total do SF-36 no grupo experimental quando comparado com o grupo controle.

Legenda: NW: Nintendo Wii. WBE: Water-based exercise. LBE: Land-based exercise. HBRT: Horseback Riding Therapy. CP: Conventional Physiotherapy. TR: Robotic Training. RITH: Rehabilitation and physical therapy. Fonte: Conceição, Conceição e Pimentel (2021).

O (Quadro 1) permite observar os impactos causados na QV de indivíduos que são acometidos por AVC, possibilitando mostrar também as principais intervenções fisioterapêuticas no manejo com essas pessoas, promovendo uma 
melhora em diversos aspectos relacionados a QV, através do questionário SF-36 que garante verificar a QV de maneira mais ampla e específica.

\section{Discussão}

Após a análise dos estudos selecionados é possível observar as repercussões dos impactos causados devido ao acometimento do AVC na QV dos indivíduos afetados, acarretando diversas complicações em diversos aspectos relacionado a saúde, principalmente nos aspectos físicos e emocionais. Diante disso, discutir sobre AVC, qualidade de vida, instrumento avaliativo e atuação da fisioterapia que é essencial para a literatura.

Segundo Santos e Waters (2020) o AVC é caracterizado como uma questão de saúde pública mundialmente, sendo a segunda no ranking de mortalidade no Brasil. Ocasionando incapacidades físicas temporárias ou definitivas, e elevando o número de internações nos leitos hospitalares. Por esse motivo surge o questionamento sobre como a qualidade de vida dessas pessoas acometidas por AVC, devidos as possíveis sequelas que as pessoas podem adquirirem, sendo motoras ou cognitivas.

Existem diversos instrumentos validados que avaliam a qualidade de vida de indivíduos que atingidos por alguma patologia, e um deles é o SF-36, este instrumento tem sido utilizado em diversos estudos para avaliação da QV, inclusive em pós-AVC. Esses instrumentos para avaliação da QV podem possibilitarem as detecções das alterações na condição de saúde, além de avaliar o prognóstico, os riscos e benefícios de determinada intervenção terapêutica. O SF-36 é composto por oito domínios: funcionamento físico, função física, dor corporal, saúde geral, vitalidade, funcionamento social, papel emocional e saúde mental. As análises componentes mostraram que existem dois conceitos distintos medidos pelo SF-36, uma dimensão física representada pelo Resumo do Componente Físico (PCS), e uma dimensão mental representada pelo Resumo do Componente Mental (MCS). Todas os domínios contribuem em diferentes proporções para a pontuação das medidas de PCS e MCS (Yang et al., 2019).

Ribeiro et al., (2015) usaram o nintendo wii no tratamento de pacientes pós AVC, após a aplicação do SF-36 eles chegaram na conclusão de que houve uma diferença significativa dentro de ambos os grupos em relação aos seguintes domínios: funcionamento físico, limitação de funções devido a aspectos físicos, vitalidade e limitação de funções devido a aspectos emocionais. E no estudo Adriani et al., (2017) também fizeram uso da mesma terapia, observaram que os pacientes tiveram aprendizado motor, obtendo um benefício sustentado através da técnica e mostraram melhora na qualidade de vida nos aspectos de capacidades funcionais. No estudo de Beinotti et al., (2013) aplicaram terapia de equitação no grupo experimental e fisioterapia convencional no grupo controle, eles verificaram que no grupo experimental houve melhora nos domínios: capacidade funcional, aspectos físicos e saúde mental.

Segundo Jacob (2012) em seu estudo com 46 idosos com sequelas de AVC, demostrou que a fisioterapia através da facilitação motora e dos treinos de atividades de vida diária garante uma melhora referente à capacidade funcional. Além da melhoria da funcionalidade dos utentes, observados, não se pode esquecer que os profissionais destas equipes facilitam a integração do utente na comunidade.

Eyvaz et al., (2018) aplicaram a terapia WBE três vezes por semana e mais o LBE duas vezes por semana no grupo de estudo e no grupo somente LBE foram cinco vezes por semana, após concluírem o período do tratamento estilado, eles verificaram que os ambos os grupos demostraram melhoras em todos os parâmetros (exceto SF-36 parâmetro dor), eles viram também que o grupo de estudo apresentou uma diferença significativa no parâmetro de vitalidade comparado ao grupo controle. Kim et al., (2015) verificaram no seu estudo que o grupo terapêutico de Tai Chi também mostrou uma melhora significativa no quesito vitalidade e em outros quatros itens (função física, dor, saúde geral, saúde mental) entre os oito itens do SF-36. 
Referente ao componente de saúde física (SF-36) dois estudos mostraram evoluções após as terapias aplicadas nos seus respectivos grupos de estudo. No estudo de Timmermans et al., (2014) foi realizado um treinamento braço-mão orientado para a tarefa, assistido por robô (grupo experimental) ou não-robótico orientado para a tarefa treinamento braço-mão (grupo controle). E já na pesquisa de Gordon et al., (2015) o grupo de estudo realizou caminha de 30 minutos por 3 semanas, é observar que os objetivos de ambos os estudos são distintos, mas conseguiram obter um mesmo resultado, ou seja, promovendo melhora no domínio de saúde física SF-36 nos grupos estudados.

Dundar et al., (2014) efetuaram terapia de treino robótico no grupo experimental, e a fisioterapia convencional no grupo controle. Por se tratar de intervenções distintas eles verificaram que ambas as terapias obtiveram melhoras significativas em todas as subpartes da SF-36. Apesar de serem técnicas diferentes, as intervenções adotadas pelos fisioterapeutas, tiveram resultados surpreendentes proporcionando melhorias em todos os domínios do instrumento SF-36. Nos achados de Liria et al., (2016) o grupo que realizou fisioterapia em casa, tiveram resultados melhores em recuperação que os pacientes que receberam reabilitação hospitalar. Os pacientes que ficaram em casa alcançaram pontuações mais altas nos domínios da funcionalidade.

No estudo de Carregosa et al., (2017) aplicaram a terapia de reabilitação virtual através do Nintendo Wii em pacientes pós AVC. O estudo mostra uma melhoria da função motora membro superior, dois meses depois, os pacientes mostraram continuidade nos aspectos funcionais e na qualidade de vida obtidas com o tratamento realizado. Já na pesquisa de Cruz et al., (2020) eles investigaram a eficácia de um programa de terapia aquática, um grupo recebeu intervenções aquática, e o outro grupo que receberam terapia em terra firme. O grupo que recebeu intervenção aquática teve resultados positivos, comparada com a terapia em terra firme.

Nos achados de Dezincourt et al., (2021) eles viram que a massoterapia aliada ao tratamento medicamentoso promove uma melhor qualidade de vida, promovendo o relaxamento muscular, com ganhos de amplitude de movimentos articulares, melhorando a coordenação dos membros afetados pela hemiparesia e nos aspectos comportamentais. Apesar do grande número de limitações físicas e cognitivas após o evento, observaram a necessidade de uma equipe multidisciplinar no acompanhamento da paciente.

Assis et al., (2021) observaram em sua pesquisa uma alta frequência de hipertensão arterial, não tendo nenhuma relação desta com nenhum dos subtipos do AVC isquêmico. Mas identificaram que o infarto parcial da circulação anterior (PACI) foi o subtipo do AVC isquêmico mais frequente e que estava associado com a presença do diabetes mellitus. Eles garantem que a coexistência de fatores de risco para os subtipos do AVC, como o diabetes mellitus e a hipertensão arterial como observado no seu estudo, é compatível com os dados da literatura. Já no estudo de Barbosa et al., (2021) verificaram uma predominância do sexo masculino, a principal faixa etária acometida em ambos os sexos, foi entre 70 e 79 anos. A morbidade hospitalar, bem como o índice de mortalidade mostrou-se elevada em pacientes com idade superior aos 80 anos, assim como o custo total de internações nessa mesma faixa etária.

No estudo de Gonçalves et al., (2018) a terapia de RV combinada com a convencional a reabilitação também mostraram um impacto positivo na função motora e destreza do membro superior, podendo ser considerado uma terapia adjuvante importante para o tratamento de alterações clínicas que afetam a dependência e comprometem a QV dos pacientes com AVC. Dessa forma, a RV mostra ter um impacto positivo na neuroplasticidade do cérebro, levando à redução da deficiência motora e proporcionando uma melhora na QV.

Apenas um estudo não mostrou um resultado positivo segundo o questionário SF-36, foi o estudo de Kiingi et al., (2017) nesse estudo eles obtiveram melhoras apenas nos domínios de visão e linguagem, mas encontram uma correlação positiva entre reintegração na comunidade e satisfação com serviços fisioterapêuticos prestados aos pacientes.

Por meio dos estudos analisados, foi possível constatar que a fisioterapia desempenha um papel importantíssimo em indivíduos diagnosticados com AVC, e que através do instrumento validado SF-36 é possível aumentar ainda mais essa 
confiabilidade, pois por meio dos dados obtidos e interpretados dos estudos a fisioterapia promove a qualidade de vida quando não em todos, mas em quase todos os aspectos avaliados.

\section{Considerações Finais}

$\mathrm{O}$ presente estudo possibilitou analisar a $\mathrm{QV}$ dos indivíduos e os principais elementos afetados devido aos diversos problemas obtidos em decorrência de um AVC. Diante disso, foi possível observar os impactos causados pelo AVC na QV dos indivíduos acometidos, acarretando complicações nos aspectos relacionados a saúde física e mental, principalmente os aspectos físicos e emocionais, foram os mais afetados e os que mais geram complicações na QV de pacientes diagnosticados com AVC. O instrumento de estudo utilizado foi o SF-36, trata-se de um questionário validado composto por oito domínios com a finalidade de avaliar e compreender a QV de pessoas que apresentam alguma complicação que venha afetar diretamente a QV, sendo a QV um principal determinantes da saúde, é necessário que tenha um olhar mais profundo com novas abordagens de pesquisas sobre o tema proposto.

Desse modo, se faz necessário a produção de novas pesquisas voltadas para avaliação da QV em indivíduos atingidos por AVC, verificando os principais aspectos afetados que interferem diretamente na $\mathrm{QV}$, podendo assim garantir com as novas pesquisas, a elaboração de estratégias específicas, com a finalidade de minimizar os impactos causados e proporcionar uma melhor QV para as pessoas acometidas.

\section{Referências}

Assis, G. L. C. B. A., Batista, S. J., Assis, T. M. R., Lima, L. R. F., França, N. M. A., Pinheiro, G. S., \& Lima, E. O. (2021). Análise do perfil dos subtipos clínicos de Doenças Cerebrovasculares Isquêmicas em pacientes admitidos em Unidade Ambulatorial em Salvador-Brasil. REAS. Vol.13(2).

Barbosa, A. M. L., Pereira, C. C. M., Miranda, J. P. R., Rodrigues, J. H. L., Carvalho, J. R. O., \& Rodrigues, A. C. E. (2021). Perfil epidemiológico dos pacientes internados por acidente vascular cerebral no nordeste do Brasil. REAS/EJCH. Vol.13(1): e5155.

Beinotti, F., Christofoletti G., Correia N., \& Borges G. (2013). Efeitos da terapia de equitação sobre Qualidade de Vida em Pacientes Pós-AVC.

Carregosa, A. A., Santos, L. F., Marcelo, R., Coêlho, M. L. S., Machado, T. C., Souza, D. C. B., Passos, G. L., Fonseca, E. P., Silva, N. M., Ribeiro, N. M. S., \& Melo, A. S. (2017). Virtual Rehabilitation through Nintendo Wii in Poststroke Patients: Follow-Up.

Carvalho, V. P., Ribeiro, H. L. S., Rocha, B. V. E., Barcelos, K. A., Andrade, F. V., Vasconcelos, G. R., Justi, J., \& Júnior, J. P. M. (2019). Perfil clínicoepidemiológico de pacientes com acidente vascular cerebral. Revista Saúde e Desenvolvimento, Vol.13(15), 50-61.

Coradini J. S., Pereira, V. C., Machado, K. F.C., Rangel, R. F., \& ILHA S. (2020). Protocolo clínico para acidente vascular cerebral: desenvolvimento de um instrumento informativo. Research, Society and Development, 9(6) e16963211.

Cruz, S. P. (2020). Influence of an Aquatic Therapy Program on Perceived Pain, Stress, and Quality of Life in Chronic Stroke Patients: A Randomized Trial.

Dezincourt, T. C. A., Dezincourt, T. M. S., Siqueira, J. P., Nascimento, F. C., Santos, K. T., Pinto, L. R., Silva, B. L., Marinho, M. V. S., Aguiar, F. P., \& Ramos, E. C. P. (2021). A prática de terapias alternativas/complementares em adultos pós acidente vascular cerebral em hemisfério esquerdo: relato de experiência. REAS, Vol. 13(3).

Dundar, U., Toktas, H., Solak, O., Ulasli, A. M., \& Eroglu, S. (2014). Estudo comparativo de fisioterapia convencional versus Treinamento robótico combinado com fisioterapia em pacientes com AVC.

Eyvaz, N., Dundar, U., \& Yesil, H. (2018). Effects of water-based and land-based exercises on walking and balance functions of patients with hemiplegia. $1053-8135 / 18 / \$ 35.00$ (C).

Gonçalves, M. G., Piva, M. F. L., Marques, C. L. S., Costa, R. D. M., Bazan, R., Luvizutto, G. J., \& Betting, L. E. G. G. (2018). Efeitos da terapia de realidade virtual na função do membro superior após AVC e o papel da neuroimagem como preditor de melhor resposta. Arq. Neuropsiquiatr. 76(10): 654-662.

Gordon, C. D., Wilks, R., \& Mccaw-Binns, A. (2015). Efeito do treinamento de exercício aeróbico (caminhada) no funcional Status e qualidade de vida relacionada à saúde em casos crônicos Sobreviventes de AVC: um ensaio clínico randomizado. Bibliotecas da Universidade de Manitoba.

Jacob, S. G. (2012). Avaliação dos cuidados de Fisioterapia domiciliária em idosos vítimas de acidente vascular cerebral. Rev. Bras. Fisioter. 12(6): 11471153.

Kiingi, A. K., Maleka, D., \& Ntsiea, V. (2017). Stroke survivors' levels of community reintegration, quality of life, satisfaction with the physiotherapy services and the level of caregiver strain at community health centres within the Johannesburg área. African Journal of Disability ISSN: (Online) 2226-7220, (Print) 2223-9170. 
Research, Society and Development, v. 10, n. 14, e506101422746, 2021

(CC BY 4.0) | ISSN 2525-3409 | DOI: http://dx.doi.org/10.33448/rsd-v10i14.22746

Kim, H. Y., Kim, Y. L., \& Lee, S. M. (2015). Effects of therapeutic Tai Chi on balance, gait, and quality of life in chronic stroke patients. Copyright $\odot$. Wolters Kluwer Health, Inc.

Liria, R. L. Ramirez, F. A. V., Perez, P. R., Parra, J. M. A., \& Gongora, D. P. (2016). Comparison of Two Post-Stroke Rehabilitation Programs: A Follow-Up Study among Primary versus Specialized Health Care.

Mello, G. A. M., Bridi, B. P. L., Oliveira, D. C., \& Jantsch, L. B. (2020). Prevalência de internações hospitalares por acidente vascular cerebral em crianças e adolescentes. Research, Society and Development, 9(7) e452974404.

Ribeiro, N. M. S., Ferraz, D. D., Pedreira, E., Pinheiro, I., Pinto, A. C. S., Neto, M. G., Santos, L. R. A., Pozzato, M. G. G., Pinho, R. S., \& Masruha, M. R. (2015). Virtual rehabilitation via Nintendo Wiit and conventional physical therapy effectively treat post-stroke hemiparetic patients. Topics in Stroke Rehabilitation, VoL. 22 NO. 4299.

Santos, L. B., \& Waters, C. (2020). Perfil epidemiológico dos pacientes acometidos por acidente vascular cerebral: revisão integrativa. Brazilian Journal of Development, 6(1), 2749-2775.

Silva, D. N., Melo, M. F. X, Duarte, E. M. M, \& Borges, A. K. P. (2019). Cuidados de enfermagem à vítima de acidente vascular cerebral (AVC): Revisão integrativa. Revista Eletrônica Acervo Saúde, 36, e2136.

Soares, C. B., Hoga, L. A. K., Peduzzi, M., Sangaleti, C., Yonekura, T., \& Silva, D. R. A. D. (2014). Revisão integrativa: conceitos e métodos utilizados na enfermagem. Rev. da Esc. Enferm. da USP, 48(2): 335-345.

Timmermans, A. A. A., Lemmens, R. J. M., Monfrance, M., Geers, R. P. J., Bakx, W., Smeets, R. J., \& Seelen, H. A. M. (2014). Efeitos do treinamento do robô orientado para a tarefa no braço função, atividade e qualidade de vida em casos crônicos pacientes com AVC: um ensaio clínico randomizado. Journal of NeuroEngineering and Rehabilitation.

Yang, F., Wong, C. K. H., Luo, N., Piercy, J., \& Jackson, J. (2019). Mapeamento da qualidade de vida da doença renal pesquisa de 36 itens (KDQOL-36) para o EQ-5D-3L e o EQ-5D-5L em pacientes submetidos a diálise. Eur J Health Econo. 8: 1195-206. 